\title{
Liraglutide and Glycaemic Outcomes in the LEADER Trial
}

Bernard Zinman · Michael A. Nauck $\cdot$ Heidrun Bosch-Traberg •

Helle Frimer-Larsen · David D. Ørsted · John B. Buse •

The LEADER Publication Committee on behalf of the LEADER Trial Investigators

Received: September 13, 2018 / Published online: November 3, 2018

(C) The Author(s) 2018

\section{ABSTRACT}

Introduction: The LEADER trial was a cardiovascular $(\mathrm{CV})$ outcomes trial in patients with type 2 diabetes at high $\mathrm{CV}$ risk that compared liraglutide $(n=4668)$ with placebo $(n=4672)$ using a primary composite endpoint of 3-point major adverse CV events. The objective of this post hoc analysis was to investigate glycaemic outcomes across both treatment groups.

Methods: Glycated haemoglobin $\left(\mathrm{HbA}_{1 \mathrm{c}}\right)$ was measured at randomisation, month 3 , month 6

Enhanced digital features To view enhanced digital features for this article go to https://doi.org/10.6084/ m9.figshare.7224140.

Electronic supplementary material The online version of this article (https://doi.org/10.1007/s13300018-0524-z) contains supplementary material, which is available to authorized users.

B. Zinman $(\bowtie)$

Lunenfeld-Tanenbaum Research Institute, Mt. Sinai

Hospital, University of Toronto, Toronto, ON,

Canada

e-mail: zinman@lunenfeld.ca

M. A. Nauck

Division of Diabetology, St. Josef-Hospital (Ruhr

University), Bochum, Germany

H. Bosch-Traberg · H. Frimer-Larsen · D. D. Ørsted

Novo Nordisk A/S, Søborg, Denmark

J. B. Buse

University of North Carolina School of Medicine,

Chapel Hill, NC, USA and every 6 months thereafter. Cox regression was used to analyse time to a composite endpoint of glycaemic deterioration, defined as a specified change in $\mathrm{HbA}_{1 \mathrm{c}}$ or a substantial intensification of insulin or oral antihyperglycaemic drug (OAD). The individual components of the composite were also analysed.

Results: Baseline characteristics, including insulin and OAD use, were balanced between treatment groups. $\mathrm{HbA}_{1 \mathrm{c}}$ decreased from baseline in both groups, but the reduction was greater with liraglutide [estimated treatment difference at month 36 : $-0.40 \%$; $95 \%$ confidence interval (CI) $-0.45,-0.34$ ] despite the addition of more OADs and higher insulin use in the placebo group. Fewer of the patients treated with liraglutide $(n=3202,68.6 \%)$ experienced glycaemic deterioration compared with those administered the placebo $(n=3988$, 85.4\%; average hazard ratio: 0.50 ; $95 \%$ CI 0.48 , $0.53 ; p<0.001)$. Analysis of the individual components showed similar results (both $p$ $<0.001)$.

Conclusions: Type 2 diabetes patients at high risk of $\mathrm{CV}$ events who were treated with liraglutide achieved greater reductions in $\mathrm{HbA}_{1 \mathrm{c}}$, had a lower risk of hypoglycaemia and presented less glycaemic deterioration than similar patients who received the placebo. Nonetheless, progressive loss of glycaemic control occurred in both groups.

Trial Registration: ClinicalTrials.gov, NCT011 79048. 
Funding: Novo Nordisk.

Plain Language Summary: Plain language summary available for this article.

Keywords: GLP-1 agonist; Glycaemic control; Incretin therapy

\section{PLAIN LANGUAGE SUMMARY}

In patients with type 2 diabetes, blood sugar levels are higher and vary more than in healthy people. In the past, some drugs that were used to treat diabetes by controlling blood sugar levels increased the risk of certain complications. LEADER was a clinical trial investigating the impact of a drug called liraglutide on stroke, heart attack and death in patients with type 2 diabetes. Liraglutide was compared with a placebo control drug, but all patients were also allowed to use a range of other diabetes drugs during the trial if needed to achieve good blood sugar control. LEADER showed that liraglutide reduced the risk of the key complications compared with placebo. While LEADER was a safety study, other variables were also measured. We examined both the haemoglobin A1C (a measure of average blood sugar levels) and the need for additional diabetes drugs as measures of how well liraglutide controlled blood sugar levels. We showed that fewer patients treated with liraglutide experienced a worsening of blood sugar control or needed additional diabetes drugs to control blood sugar. This means that patients treated with liraglutide had better blood sugar control over time than those who received the placebo. While blood sugar control worsened with both placebo and liraglutide over the 5 years of the study, this worsening was faster with the placebo.

\section{INTRODUCTION}

Glycaemic control is an important factor in the development of diabetes-related complications [1-3]. Glucagon-like peptide-1 receptor agonists (GLP-1RAs) have demonstrated efficacy in glycaemic control in patients with type 2 diabetes [4]; however, for many of these agents, data on glycaemic control beyond 2 years are lacking. Prior to the publication of the results from the Liraglutide Effect and Action in Diabetes: Evaluation of Cardiovascular Outcome Results (LEADER) trial, the same was true for liraglutide, with few long-duration trials $[5,6]$. LEAD-2 [6] and LEAD-3 [5] demonstrated the efficacy of liraglutide $1.8 \mathrm{mg}$ over 2 years (mean change from baseline in glycated haemoglobin $\left[\mathrm{HbA}_{1 \mathrm{c}}\right]$ : $-0.6 \%$ and $-1.1 \%$, respectively).

Analysis of the data in the LEADER trial demonstrated a significant reduction in the primary endpoint (3-point major adverse cardiovascular [CV] events [MACE]: the first occurrence of $\mathrm{CV}$ death, nonfatal myocardial infarction or nonfatal stroke) with liraglutide versus placebo, both on a background of standard-of-care treatment [7]. Blood glucose levels and other laboratory parameters were measured regularly during the trial [7]. An $\mathrm{HbA}_{1 \mathrm{c}}$ level of $\leq 7.0 \%$ (individualised depending on the patient) was targeted, modified according to local guidelines [7]. While Marso et al. analysed the change in $\mathrm{HbA}_{1 \mathrm{c}}$ levels from baseline to month 36 of the trial [7], a more detailed analysis of these data has not yet been published.

The objective of this post hoc analysis of the LEADER trial is to describe glycaemic effectiveness and durability with liraglutide compared with placebo during a follow-up period of up to 5 years in patients with type 2 diabetes at high CV risk.

\section{METHODS}

LEADER (NCT01179048) was a double-blind, placebo-controlled, event- and time-driven trial with a follow-up period ranging from 42 to 60 months. Patients were randomly assigned $1: 1$ to receive liraglutide $1.8 \mathrm{mg}$ (or the maximum tolerated dose) or placebo once daily, both in addition to standard-of-care treatment [7]. Standard-of-care treatment included stressing the importance of all aspects of lifestyle management for glycaemic control. If $\mathrm{HbA}_{1 \mathrm{c}}$ remained $>7 \%$ (or the individualised target) with trial intervention, investigators were encouraged to intensify treatment by initiating or increasing the dose of any 
antihyperglycaemic therapy (except dipeptidyl peptidase-4 [DPP4] inhibitors, other GLP-1RAs or pramlintide) $[7,8]$. Informed consent was obtained from all participants in the LEADER study. All procedures performed in this study were in accordance with the ethical standards of the local research committees and with the 1964 Declaration of Helsinki and its later amendments [7].

The LEADER trial design strived for glycaemic equipoise across treatment groups, consistent with $\mathrm{CV}$ outcomes trial (CVOT) protocols for diabetes therapies. This design was intended to diminish the potential effects of glycaemic control on CV outcomes. To evaluate this outcome, $\mathrm{HbA}_{1 \mathrm{c}}$ was measured at randomisation, month 3 , month 6 and then every subsequent 6 months [7]. The changes in $\mathrm{HbA}_{1 \mathrm{c}}$ over time and at 36 months have been published [7] but have not been analysed in detail.

Hypoglycaemia events were captured as safety endpoints, where severe events were those requiring assistance from another person, and confirmed events were those with plasma glucose levels $<3.1 \mathrm{mmol} / \mathrm{L}[7,8]$.

\section{Analysis}

A composite endpoint was used to assess glycaemic deterioration. It was defined as $\mathrm{HbA}_{1 \mathrm{c}}$ $\geq 8.0 \%$ and reduction in $\mathrm{HbA}_{1 \mathrm{c}}<0.5 \%$ since the previous visit, or substantial intensification of insulin or oral antihyperglycaemic drug (OAD; start of new OAD or start of insulin or increase of insulin dose $\geq 10$ units [U] or addition of mealtime bolus to basal insulin or a shift from basal insulin to premixed insulin). The $\mathrm{HbA}_{1 \mathrm{c}}$ component of this endpoint was designed to capture:

(a) The non-attainment of an $\mathrm{HbA}_{1 \mathrm{c}}$ target ( $\leq 7.0 \%$ or individualised for each patient at the discretion of the investigator); to simplify this measure, and based on the high mean baseline $\mathrm{HbA}_{1 \mathrm{c}}$ value of $8.7 \%$, a uniform value of $8.0 \%$ was selected for this analysis

(b) Events where therapy no longer reduced $\mathrm{HbA}_{1 \mathrm{c}}(<0.5 \%)$ in patients with $\mathrm{HbA}_{1 \mathrm{c}}$ above the target.
With respect to changes in insulin dose, due to the level of data available, a $10 \mathrm{U}$ increase in insulin was chosen as a threshold that balances sensitivity and specificity for deterioration in glycaemic control.

The Cox regression method was used to analyse time to glycaemic deterioration in liraglutide-treated versus placebo-treated patients. Time to the individual components of this composite endpoint was also examined using the Cox regression method.

Cumulative incidence plots were used to show the time to glycaemic deterioration, and were estimated using the Aalen-Johansen method with death as a competing risk factor. Death was investigated as a competing risk factor to negate the effect of the treatment imbalance in patients who died before glycaemic deterioration occurred (greater CV mortality in the placebo group compared with liraglutide [7]). The Weibull model was then used to estimate the average event time ratio. The degree of deviation from proportional hazards was investigated by fitting the Weibull model by treatment, which allowed the calculation of the hazard ratio (HR) over time and quartiles of event time ratios.

Changes from baseline in $\mathrm{HbA}_{1 \mathrm{c}}$ were analysed by baseline $\mathrm{HbA}_{1 \mathrm{c}}$ quartiles. Upon analysing the baseline $\mathrm{HbA}_{1 \mathrm{c}}$ in all patients, four quartiles were identified: patients with $\mathrm{HbA}_{1 \mathrm{c}}<7.6 \%, \quad \mathrm{HbA}_{1 \mathrm{c}} \geq 7.6 \%$ and $<8.3 \%$, $\mathrm{HbA}_{1 \mathrm{c}} \geq 8.3 \%$ and $<9.4 \%$, and $\mathrm{HbA}_{1 \mathrm{c}} \geq 9.4 \%$, respectively.

The rate ratios of confirmed and severe hypoglycaemia events were estimated using a negative binomial regression using a log link and the logarithm of the observation time as offset.

\section{RESULTS}

Baseline characteristics were balanced between the two treatment groups (liraglutide $n=4668$; placebo $n=4672$ ), with a mean age of 64 years, diabetes duration of 12.8 years and $\mathrm{HbA}_{1 \mathrm{c}}$ of $8.7 \%$ [7]. The proportions of patients using OADs only, insulin only or both were also similar at baseline in the two treatment groups 
Table 1 Baseline characteristics of the liraglutide and placebo groups, including concomitant antihyperglycaemic treatment

\begin{tabular}{|c|c|c|}
\hline & Liraglutide $(n=4668)$ & Placebo $(n=4672)$ \\
\hline Male sex ${ }^{a}$ & $3011(64.5)$ & $2992(64.0)$ \\
\hline Age, years ${ }^{\mathrm{a}}$ & $64.2 \pm 7.2$ & $64.4 \pm 7.2$ \\
\hline Diabetes duration, years ${ }^{a}$ & $12.8 \pm 8.0$ & $12.9 \pm 8.1$ \\
\hline \multicolumn{3}{|l|}{ Geographic region ${ }^{\mathrm{a}}$} \\
\hline Europe & $1639(35.1)$ & $1657(35.5)$ \\
\hline North America & $1401(30.0)$ & $1446(31.0)$ \\
\hline Asia & $360(7.7)$ & $351(7.5)$ \\
\hline Rest of the world & $1268(27.2)$ & $1218(26.1)$ \\
\hline $\mathrm{HbA}_{1 \mathrm{c}}, \%^{\mathrm{a}}$ & $8.7 \pm 1.6$ & $8.7 \pm 1.5$ \\
\hline BMI, $\mathrm{kg} / \mathrm{m}^{2}$ a & $32.5 \pm 6.3$ & $32.5 \pm 6.3$ \\
\hline Body weight, $\mathrm{kg}^{\mathrm{a}}$ & $91.9 \pm 21.2$ & $91.6 \pm 20.8$ \\
\hline Systolic blood pressure, $\mathrm{mm} \mathrm{Hg}^{\mathrm{a}}$ & $135.9 \pm 17.8$ & $135.9 \pm 17.7$ \\
\hline Diastolic blood pressure, $\mathrm{mm} \mathrm{Hg}^{\mathrm{a}}$ & $77.2 \pm 10.3$ & $77.0 \pm 10.1$ \\
\hline Established CVD/chronic kidney disease (age $\geq 50)^{\mathrm{a}, \mathrm{b}}$ & $3831(82.1)$ & $3767(80.6)$ \\
\hline CVD risk factors $(\text { age } \geq 60)^{a, c}$ & $837(17.9)$ & $905(19.4)$ \\
\hline \multicolumn{3}{|l|}{ Antihyperglycaemic medication at baseline } \\
\hline OAD only & $2436(52.2)$ & $2375(50.8)$ \\
\hline $1 \mathrm{OAD}$ & $916(19.6)$ & $894(19.1)$ \\
\hline 2 OADs & $1357(29.1)$ & $1321(28.3)$ \\
\hline$>2 \mathrm{OADs}$ & $163(3.5)$ & $160(3.4)$ \\
\hline Insulin only & $361(7.7)$ & $377(8.1)$ \\
\hline Insulin + OAD & $1677(35.9)$ & $1754(37.5)$ \\
\hline Insulin + metformin & $1397(29.9)$ & $1500(32.1)$ \\
\hline None & $194(4.2)$ & $166(3.6)$ \\
\hline Insulin dose, $U$ & $51.5 \pm 43.0$ & $51.9 \pm 44.1$ \\
\hline Per body weight, $\mathrm{U} / \mathrm{kg}$ & $0.55 \pm 0.41$ & $0.55 \pm 0.39$ \\
\hline
\end{tabular}

Full analysis set. Data are the mean \pm standard deviation or the number of patients (percentage of either liraglutide or placebo group). Percentage data refer to the proportion of patients

${ }^{a}$ Data first published in the LEADER primary publication (Marso et al. [7])

b Established CVD was defined as prior myocardial infarction or prior stroke or transient ischaemic attack or prior revascularisation or $>50 \%$ stenosis of coronary, carotid or lower extremity arteries or documented symptomatic coronary heart disease or documented asymptomatic cardiac ischaemia or heart failure or chronic kidney disease

c CVD risk factors included microalbuminuria or proteinuria, hypertension and left ventricular hypertrophy, left ventricular systolic or diastolic dysfunction, or ankle-brachial index $<0.9$.

$B M I$ body mass index, $C V D$ cardiovascular disease, $H b A_{1 c}$ glycated haemoglobin, $O A D$ oral antihyperglycaemic drug 
Table 2 Estimated risk of glycaemic deterioration over 5 years with liraglutide versus placebo

\begin{tabular}{llll}
\hline Endpoint & $\begin{array}{l}\text { Liraglutide, } \\
\boldsymbol{n}(\%)\end{array}$ & $\begin{array}{l}\text { Placebo, } \\
\boldsymbol{n}(\%)\end{array}$ & $\begin{array}{l}\text { Average hazard ratio [95\% CI], } \\
\boldsymbol{p} \text { value }\end{array}$ \\
\hline $\begin{array}{l}\text { Glycaemic deterioration } \\
{ }^{\mathrm{a}}\end{array}$ & $3202(68.6)$ & $3988(85.4)$ & $0.50[0.48 ; 0.53], p<0.001$ \\
$\mathrm{HbA}_{\mathrm{lc}} \geq 8.0 \%$ and reduction $<0.5 \%$ & $2486(53.3)$ & $3410(73.0)$ & $0.50[0.47 ; 0.52], p<0.001$ \\
$\begin{array}{l}\text { Substantial intensification of insulin or } \\
\mathrm{OAD}^{\mathrm{b}}\end{array}$ & $2267(48.6)$ & $3046(65.2)$ & $0.59[0.56 ; 0.62], p<0.001$ \\
\hline
\end{tabular}

Cox regression of time to the composite endpoint

${ }^{a}$ Glycaemic deterioration defined as $\mathrm{HbA}_{1 \mathrm{c}} \geq 8.0 \%$ and reduction $<0.5 \%$ since previous visit, or substantial intensification of insulin or OAD

b Substantial intensification of insulin or OAD defined as: start of new OAD; start of insulin; increase in insulin dose $\geq 10$ $\mathrm{U}$; or addition of mealtime bolus insulin to basal insulin or a shift from basal insulin to premixed insulin.

$C I$ confidence interval, $H b A_{1 c}$ glycated haemoglobin, $O A D$ oral antihyperglycaemic drug

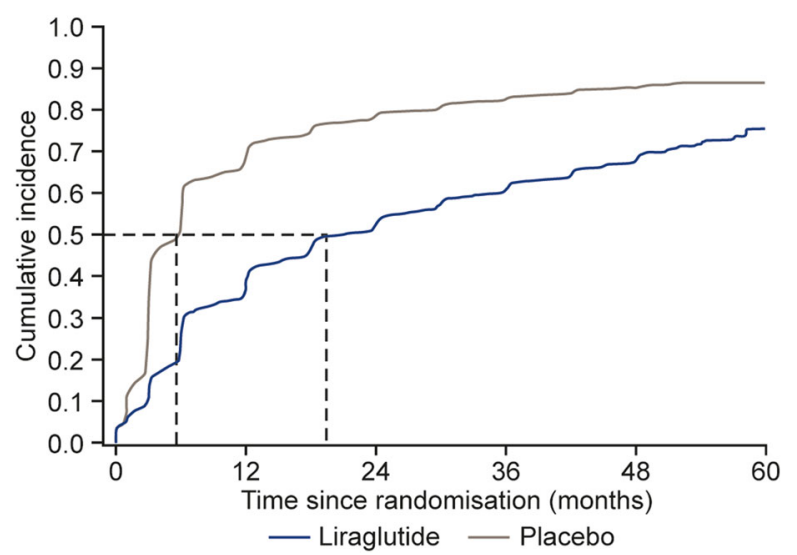

Fig. 1 Cumulative incidence plot of time to $\mathrm{HbA}_{1 \mathrm{c}} \geq 8 \%$ and reduction $<0.5 \%$ since previous visit or substantial intensification* in insulin or OAD treatment. Aalen-Johansen plot, with death as a competing risk factor. *Substantial intensification of insulin or OAD defined as: start of new OAD; start of insulin; increase in insulin dose $\geq 10$ units; or addition of mealtime bolus insulin to basal insulin or a shift from basal insulin to premixed insulin. $H b A_{1 c}$ glycated haemoglobin, $O A D$ oral antihyperglycaemic drug

(Table 1), and a total of $55 \%$ of patients were insulin naïve. Of patients who were using insulin at baseline, the mean insulin dose in both groups was similar in terms of overall dose and weight-normalised dose (Table 1).

Mean $\mathrm{HbA}_{1 \mathrm{c}}$ decreased from baseline with both liraglutide and placebo. Previously, we reported that this reduction was greater with liraglutide [mean change at month $36:-1.16 \%$ with liraglutide versus $-0.77 \%$ with placebo; estimated treatment difference at month 36: - 0.40\%; 95\% confidence interval (CI) -0.45 , - 0.34] [7]. This was despite a greater use of additional glucose-lowering medications in the placebo treatment group [7]. In our current, more detailed analysis, we found that of the patients in the liraglutide group, $41.6 \%$ were at an $\mathrm{HbA}_{1 \mathrm{c}}$ target of $<7 \%$ at month 36 , compared with $25.8 \%$ in the placebo group. Analysis of the change in $\mathrm{HbA}_{1 \mathrm{c}}$ from baseline, according to baseline $\mathrm{HbA}_{1 \mathrm{c}}$ quartiles, demonstrated that patients with the highest baseline $\mathrm{HbA}_{1 \mathrm{c}}$ measurement had the largest reductions over the course of the trial (Fig. S1 in the Electronic supplementary material, ESM). Differences between the liraglutide and placebo groups within these four quartiles were largest during the first 12-18 months of the trial, after which the $\mathrm{HbA}_{1 \mathrm{c}}$ levels of both groups converged (Fig. S1 in the ESM).

This new analysis of the LEADER glycaemic data demonstrated that the risk of deterioration in glycaemic control was significantly larger in patients treated with the placebo $(n=3988$, 85.4\%) compared with those treated with liraglutide $(n=3202,68.6 \%$; average HR of liraglutide versus placebo: 0.50 ; $95 \%$ CI 0.48 , $0.53 ; p<0.001$; Table 2, Fig. 1 ). Fifty percent of patients in the placebo group experienced glycaemic deterioration after only 4.8 months, compared with after 19.5 months for the 
liraglutide group (Fig. 1). The estimated time to glycaemic deterioration was, on average, threefold longer with liraglutide compared with placebo (Weibull estimate: 3.57 ; 95\% CI 3.28, 3.88; $p<0.0001)$. The HR estimated from the Weibull model over time for the composite endpoint between the liraglutide and placebo groups was 0.48 (95\% CI $0.46,0.50)$ in month 3 , and increased to $0.62(95 \%$ CI $0.58,0.67)$ in month 36. Sensitivity analyses of the composite by patients who were insulin naïve at baseline or OAD naïve at baseline did not change the average HR between liraglutide and placebo (HR: 0.50; 95\% CI 0.47, 0.53; and HR: 0.51; 95\% CI $0.44,0.58$, respectively). The estimated subdistribution HR with death as a competing risk was very similar to the corresponding causespecific HR from the Cox regression (data not shown).

This current detailed analysis of the individual components of the glycaemic deterioration composite endpoint demonstrated that both the $\mathrm{HbA}_{1 \mathrm{c}}$ and the increase in insulin or OAD components were significantly different across the two treatment groups, with more patients in the placebo group failing to control $\mathrm{HbA}_{1 \mathrm{c}}$ and more requiring increases in insulin or OAD (Table 2, Fig. 2). Mean insulin dose increased in both treatment groups during the trial, and was higher in the placebo group compared with the liraglutide group, throughout the trial (Fig. S2 in the ESM). At month 36, the mean insulin dose was $49.4 \mathrm{U}(n=2010)$ in the liraglutide group and $57.5 \mathrm{U}(n=2506)$ in the placebo group. Overall, the risk of patients increasing their insulin dose by $10 \mathrm{U}$ or more was significantly higher for those in the placebo group compared with the liraglutide group (HR 0.61; 95\% CI $0.56,0.65 ; p<0.001$ ) (Fig. S3 in the ESM).

As previously reported in the primary LEADER publication, analysis of the estimated rate ratio of severe hypoglycaemic events (liraglutide/placebo) demonstrated that patients treated with liraglutide had an estimated 31\% lower rate of experiencing such events compared with the placebo treatment group (treatment rate ratio: $0.69 ; 95 \%$ CI 0.51, 0.93; $p=0.013$ ) [7]. A similar trend was apparent for confirmed hypoglycaemic events (treatment rate ratio: $0.81 ; 95 \%$ CI $0.74,0.88 ; p<0.001$ ) [7].
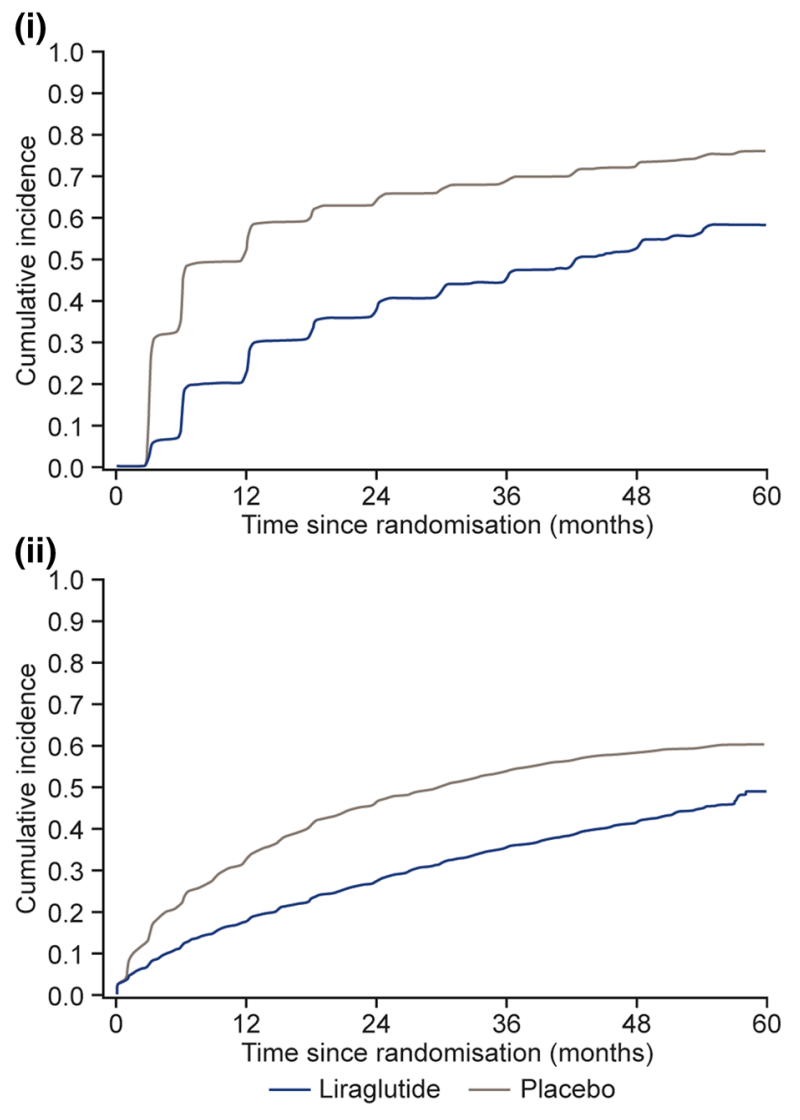

Fig. 2i-ii Cumulative incidence plots for both parts of the composite endpoint. $\mathbf{i}$ Time to $\mathrm{HbA}_{1 \mathrm{c}} \geq 8 \%$ and reduction $<0.5 \%$ since previous visit. ii Time to substantial intensification* of insulin or OAD treatment. Aalen-Johansen plots, with death as a competing risk factor. *Substantial intensification of insulin or OAD defined as: start of new $\mathrm{OAD}$; start of insulin; increase in insulin dose $\geq 10$ units; or addition of mealtime bolus insulin to basal insulin or a shift from basal insulin to premixed insulin. $H b A_{1 c}$ glycated haemoglobin, $O A D$ oral antihyperglycaemic drug

\section{DISCUSSION}

The analysis described here shows that treatment with liraglutide in the LEADER trial was associated with lower $\mathrm{HbA}_{1 \mathrm{c}}$, reduced incidence of glycaemic deterioration and delayed treatment intensification compared with placebo in patients with type 2 diabetes at high risk of $\mathrm{CV}$ disease. This was over the trial duration, which had a median follow-up time of 3.8 years [7], despite both groups aiming for local standard- 
of-care glycaemic targets. Nonetheless, both treatment groups demonstrated progressive glycaemic deterioration over the course of the study, consistent with the progressive nature of type 2 diabetes.

Long-term, clinical trial data for GLP1-RAs are relatively scant. Before LEADER, there were only two trials greater than 1 year in duration, both of which reported similar $\mathrm{HbA}_{1 \mathrm{c}}$ reductions from baseline to LEADER [5, 6] (Table S1 in the ESM and [9]). Results from 6 years of exenatide once-weekly (QW) treatment in the DURATION-1 trial, uncontrolled, extension phase $(n=136)$ reported a decrease in $\mathrm{HbA}_{1 \mathrm{c}}$ of approximately $1.6 \%$ [10]. The generalisability of this result is limited by the lack of a control group and the large number of patients who withdrew consent during the trial, with the attendant potential for selection bias whereby those with good responses to study drug likely continued and those with less benefit may have preferentially withdrawn [10]. There are also 3 -year data for exenatide across three of the DURATION trials (DURATION-1, -2 and -3 ), and pooled data from these trials have demonstrated that reductions in $\mathrm{HbA}_{1 \mathrm{c}}$ were maintained at $1.1 \%$ from baseline at year 3 [11]. Similarly, pooled data from five of the HARMONY studies have demonstrated that albiglutide reduced $\mathrm{HbA}_{1 \mathrm{c}}$ by up to $0.92 \%$ at the end of year 3 [12]. Cross-trial efficacy comparisons should always be made with caution. Such longterm data provide evidence that the safety and efficacy of GLP-1RAs reported in the first clinical trials with a duration of less than 1 year can be maintained for several years in a large number of patients.

CVOTs have also been conducted to investigate other classes of glucose-lowering drugs, allowing the long-term evaluation of glycaemic control by sodium-glucose cotransporter 2 inhibitors (SGLT-2is) and DPP-4 inhibitors [13-18]. They have also reported changes in glycaemic control, albeit not as their primary outcome. In EMPA-REG, empagliflozin $25 \mathrm{mg}$ reduced $\mathrm{HbA}_{1 \mathrm{c}}$ from baseline by $0.36 \%$ at week 206 ( 4 years) compared with placebo [13], while in CANVAS, canagliflozin reduced it by $0.58 \%$ at week 338 ( 6.5 years) [14]. For DPP4 inhibitors, sitagliptin decreased $\mathrm{HbA}_{1 \mathrm{c}}$ by $0.29 \%$ in TECOS versus placebo [15], alogliptin by $0.33 \%$ in EXAMINE [18], and saxagliptin by $0.2 \%$ in SAVOR-TIMI $53[16,17]$. Liraglutide (difference versus placebo of $-0.4 \%$; [7]) compared favourably with these other classes of glucose-lowering drugs.

Although the degree of reduction in $\mathrm{HbA}_{1 \mathrm{c}}$ from baseline observed in the LEADER trial (approximately 1\%) demonstrates the effectiveness of liraglutide in patients with advanced type 2 diabetes, the trial did reveal significant glycaemic deterioration over 5 years. This is expected with the progressive nature of type 2 diabetes and has been seen in a similar trial [17], and it occurred more frequently in the placebo than in the liraglutide group. At the end of the LEADER trial, the vital status of $99.7 \%$ of patients was known, with a total of $96.8 \%$ attending a final visit, dying or experiencing a primary outcome [7]. This high retention rate provides confidence in our results and is somewhat greater than retention rates in other trials (46-66\%), though these rates may not be directly comparable [10-12].

Though the general target for $\mathrm{HbA}_{1 \mathrm{c}}$ in the LEADER protocol was $\leq 7 \%[7,8]$; it was meant to be individualised, based on investigator discretion [8]. A change of $0.5 \% \mathrm{HbA}_{1 \mathrm{c}}$ is the generally accepted minimal clinically important difference $[19,20]$. Therefore, the composite endpoint in this analysis used an $\mathrm{HbA}_{1 \mathrm{c}}$ cutoff of $\geq 8.0 \%$ and/or a reduction in $\mathrm{HbA}_{1 \mathrm{c}}<0.5 \%$ between visits (every 6 months for most of the trial) as a composite index of glycaemic deterioration.

It is not surprising that the glycaemic effectiveness observed with liraglutide was achieved with fewer additions of glucose-lowering medications [7] and a lower insulin dose compared with the placebo group. Of particular interest, this was also associated with a reduction in severe hypoglycaemia. The LEADER trial had specific standard-of-care guidelines in which investigators were able to treat hyperglycaemia with few restrictions, except that it was recommended that the daily dose of premixed insulin was not increased during the first 2 weeks postrandomisation, and if $\mathrm{HbA}_{1 \mathrm{c}}$ was $<8 \%$, it was recommended that the daily dose was decreased by $20 \%[7,8]$. This contrasts with other CVOTs, 
such as EMPA-REG OUTCOME, where the baseline antihyperglycaemic medication had to remain unchanged for the first 12 weeks of trial participation [13]. The LEADER protocol did not allow the use of other GLP-1RAs or DPP-4 inhibitors, as these two classes overlap in mechanistic pathways with liraglutide, potentially confounding the CV outcome results of the trial. SGLT-2is were not available at the time of LEADER trial initiation, their CV risk profile was unknown when the study was conducted, and the safety of their combination with GLP1RAs had not been established. As a result, investigators were discouraged from adding SGLT-2is later, and less than 3\% of patients in both treatment groups were prescribed SGLT-2is during the trial [7].

There are limitations to these analyses. They are post hoc. The results may be affected by the use of a wide range of concomitant medications and the masking of liraglutide and placebo, but this was otherwise a relatively real-world setting reflecting how liraglutide might be used in clinical practice. The limitations of the original trial also apply, in that it was completed within a pre-specified population (i.e. patients with type 2 diabetes and at high risk for CV events) [7].

\section{CONCLUSION}

In conclusion, in the LEADER trial, patients with advanced type 2 diabetes at high CV risk treated with liraglutide experienced greater reductions in $\mathrm{HbA}_{1 \mathrm{c}}$ and a lower risk of glycaemic deterioration and hypoglycaemia when compared with patients treated with placebo, despite the increased use of other glucose-lowering medications and higher insulin doses in the placebo group. Nevertheless, both treatment groups experienced a progressive loss of glycaemic control.

\section{ACKNOWLEDGEMENTS}

Funding. Novo Nordisk funded the original LEADER study and this post hoc analysis of the results. Novo Nordisk also funded the journal's article processing charges. All authors had full access to all of the data in this study and take complete responsibility for the integrity of the data and accuracy of the data analysis.

Editorial Assistance. Editorial support was provided by Gillian Groeger, PhD, and Izabel James, MBBS, both from Watermeadow Medical, an Ashfield company, part of UDG Healthcare plc, and funded by Novo Nordisk.

Authorship. All named authors meet the International Committee of Medical Journal Editors (ICMJE) criteria for authorship for this article, take responsibility for the integrity of the work as a whole, and have given their approval for this version to be published.

Authorship Contributions. Bernard Zinman, Michael A. Nauck and John B. Buse are members of the LEADER trial steering committee, contributed to the trial design, and were involved in the design and interpretation of this post hoc analysis. Heidrun Bosch-Traberg, Helle Frimer-Larsen and David D. Ørsted designed and completed the post hoc analysis.

Prior Presentation. Parts of this analysis were presented at the International Diabetes Federation (IDF) Congress 2017, 4-8 December 2017, Abu Dhabi, United Arab Emirates.

Disclosures. Bernard Zinman has consulted for and received honoraria from AstraZeneca, Boehringer Ingelheim, Eli Lilly, Janssen, Merck, Novo Nordisk, Sanofi and Takeda; he has received grant support from Boehringer Ingelheim, Novo Nordisk and AstraZeneca. Michael A. Nauck has been a member on advisory boards or has consulted for AstraZeneca, Boehringer Ingelheim, Eli Lilly, Fractyl, GlaxoSmithKline, Menarini/Berlin Chemie, Merck, Sharp \& Dohme and Novo Nordisk; he has received grant support from Boehringer Ingelheim, Eli Lilly, GlaxoSmithKline, Merck, Sharp \& Dohme, Novartis Pharma and Novo Nordisk; he has served on the speakers' bureau of AstraZeneca, Boehringer Ingelheim, Eli Lilly, Menarini/Berlin Chemie, Merck, Sharp \& Dohme and Novo 
Nordisk. Heidrun Bosch-Traberg is an employee of and holds stock in Novo Nordisk. Helle Frimer-Larsen is an employee of and holds stock in Novo Nordisk. David D. Ørsted is an employee of and holds stock in Novo Nordisk. John B. Buse's contracted consulting fees are paid to the University of North Carolina by Adocia, AstraZeneca, Dance Biopharm, Eli Lilly, MannKind, NovaTarg, Novo Nordisk, Senseonics, vTv Therapeutics and Zafgen; he has received grant support from Novo Nordisk, Sanofi and vTv Therapeutics; he is a consultant to Neurimmune AG; he holds stock options in Mellitus Health, PhaseBio and Stability Health; and he is supported by a grant from the National Institutes of Health (UL1TR002489).

Compliance with Ethics Guidelines. Informed consent was obtained from all participants in the LEADER study. All procedures performed in this study were in accordance with the ethical standards of the local research committees and with the 1964 Declaration of Helsinki and its later amendments.

Data Availability. The datasets generated during and/or analysed during the current study are available from the corresponding author on reasonable request.

Open Access. This article is distributed under the terms of the Creative Commons Attribution-NonCommercial 4.0 International License (http://creativecommons.org/licenses/ by-nc/4.0/), which permits any noncommercial use, distribution, and reproduction in any medium, provided you give appropriate credit to the original author(s) and the source, provide a link to the Creative Commons license, and indicate if changes were made.

\section{REFERENCES}

1. International Diabetes Federation. IDF global guideline for type 2 diabetes. 2012. http://www.idf. org/sites/default/files/IDF-Guideline-for-Type-2Diabetes.pdf. Accessed 16 Feb 2017.
2. Patel A, MacMahon S, Chalmers J, et al. Intensive blood glucose control and vascular outcomes in patients with type 2 diabetes. $\mathrm{N}$ Engl J Med. 2008;358:2560-72.

3. UKPDS. Intensive blood-glucose control with sulphonylureas or insulin compared with conventional treatment and risk of complications in patients with type 2 diabetes (UKPDS 33). UK Prospective Diabetes Study (UKPDS) Group. Lancet. 1998;352:837-53.

4. Harris KB, McCarty DJ. Efficacy and tolerability of glucagon-like peptide-1 receptor agonists in patients with type 2 diabetes mellitus. Ther Adv Endocrinol Metab. 2015;6:3-18.

5. Garber A, Henry RR, Ratner R, Hale P, Chang CT, Bode B. Liraglutide, a once-daily human glucagonlike peptide 1 analogue, provides sustained improvements in glycaemic control and weight for 2 years as monotherapy compared with glimepiride in patients with type 2 diabetes. Diabetes Obes Metab. 2011;13:348-56.

6. Nauck M, Frid A, Hermansen K, et al. Long-term efficacy and safety comparison of liraglutide, glimepiride and placebo, all in combination with metformin in type 2 diabetes: 2-year results from the LEAD-2 study. Diabetes Obes Metab. 2013;15:204-12.

7. Marso SP, Daniels GH, Brown-Frandsen K, et al. Liraglutide and cardiovascular outcomes in type 2 diabetes. N Engl J Med. 2016;375:311-22.

8. Marso SP, Poulter NR, Nissen SE, et al. Design of the liraglutide effect and action in diabetes: evaluation of cardiovascular outcome results (LEADER) trial. Am Heart J. 2013;166(823-30):e5.

9. Rigato M, Fadini GP. Comparative effectiveness of liraglutide in the treatment of type 2 diabetes. Diabetes Metab Syndr Obes. 2014;7:107-20.

10. Henry RR, Klein EJ, Han J, Iqbal N. Efficacy and tolerability of exenatide once weekly over 6 years in patients with type 2 diabetes: an uncontrolled open-label extension of the DURATION-1 study. Diabetes Technol Ther. 2016;18:677-86.

11. Trautmann ME, Van Gaal L, Han J, Hardy E. Threeyear efficacy and safety of exenatide once weekly: a pooled analysis of three trials. J Diabetes Compl. 2017;31:1415-22.

12. Home PD, Ahren B, Reusch JEB, et al. Three-year data from 5 HARMONY phase 3 clinical trials of albiglutide in type 2 diabetes mellitus: long-term efficacy with or without rescue therapy. Diabetes Res Clin Pract. 2017;131:49-60. 
13. Zinman B, Wanner C, Lachin JM, et al. Empagliflozin, cardiovascular outcomes, and mortality in type 2 diabetes. N Engl J Med. 2015;373:2117-28.

14. Neal B, Perkovic V, Mahaffey KW, et al. Canagliflozin and cardiovascular and renal events in type 2 diabetes. N Engl J Med. 2017;377:644-57.

15. Green JB, Bethel MA, Armstrong PW, et al. Effect of sitagliptin on cardiovascular outcomes in type 2 diabetes. N Engl J Med. 2015;373:232-42.

16. Scirica BM, Bhatt DL, Braunwald E, et al. Saxagliptin and cardiovascular outcomes in patients with type 2 diabetes mellitus. N Engl J Med. 2013;369:1317-26.

17. Leibowitz G, Cahn A, Bhatt DL, et al. Impact of treatment with saxagliptin on glycaemic stability and beta-cell function in the SAVOR-TIMI 53 study. Diabetes Obes Metab. 2015;17:487-94.

18. White WB, Cannon CP, Heller SR, et al. Alogliptin after acute coronary syndrome in patients with type 2 diabetes. N Engl J Med. 2013;369:1327-35.

19. Internal Clinical Guidelines Team. Type 2 diabetes in adults: management. London: National Institute for Health and Care Excellence. 2015. https://www. nice.org.uk/guidance/ng28/evidence/fullguideline-pdf-78671532569. Accessed 15 Oct 2018.

20. Lenters-Westra E, Schindhelm RK, Bilo HJ, Groenier $\mathrm{KH}$, Slingerland RJ. Differences in interpretation of haemoglobin A1c values among diabetes care professionals. Neth J Med. 2014;72:462-6. 